\title{
ON A THEOREM OF ZASSENHAUS ${ }^{1}$
}

H. E. GOHEEN

In a recent paper Zassenhaus [1] has proved the following theorem: if in a finite group $G$ the normalizer of every Abelian group is also its centralizer, then $G$ is Abelian. It is the purpose of this note to give an alternative proof of this theorem.

Let $G$ be a finite group in which the normalizer of every Abelian subgroup is its centralizer. In an induction proof we assume that all groups of order less than the order of $G$ which satisfy the hypothesis of the theorem are Abelian. Then in particular all the subgroups of $G$ are Abelian.

In case the center of $G$ is not the identity, Zassenhaus has the following argument. The order of $G / Z$ is less than that of $G$. But the same hypotheses as for $G$ hold for the quotient group and, therefore, $G / Z$ is Abelian. Then if $a$ and $b$ are any two elements of $G, a^{-1} b a$ $=b(Z)$ and $a z=z a$. Thus $a$ is in the normalizer of the Abelian group $(b, Z)$ and hence, by hypothesis, commutes with everything in $(b, Z)$ including $b$. Thus $a$ and $b$ are commutative and $G$ is Abelian.

The alternative case cannot occur, for in case the center of $G$ is the identity, then either a maximal subgroup of $G$ is invariant or not. In the former case $G$ is the centralizer of a maximal invariant subgroup $G_{1}$, for such a subgroup would as noted be Abelian. But if $\rho$ is not in $G_{1},\left\{\rho, G_{1}\right\}=G$. Then, since $G_{1}$ is Abelian and $\rho$ is in the centralizer of $G_{1}$ and hence commutes with all its elements, $G$ is Abelian.

It will now be shown that $G_{1}$ cannot be noninvariant if $G$ is not Abelian and if the center of $G$ is the identity. For then the group $G$ has a representation as a permutation group on $G_{1}$ and its conjugates, an element $x$ corresponding to the permutation

$$
(x)=\left(\begin{array}{c}
G_{i} \\
x^{-1} G_{i} x
\end{array}\right)
$$

for the $n$ conjugate subgroups $G_{1}, G_{2}, \cdots, G_{n}$. The representation is 1-1 in this case; and the subgroup fixing one letter in it, say $G_{i}$, is the subgroup $G_{i}$ of $G$. If, in this subgroup, there is an element $y$ corresponding to a permutation fixing more than one letter, say $G_{j}$, as well, then $y$ is in both $G_{i}$ and $G_{j}$. Since $G_{i}$ and $G_{j}$ are both Abelian

Received by the editors January 15, 1954.

1 The author wishes to thank the editor for his assistance. 
and both maximal, then every element of the group $G=\left\{G_{i}, G_{j}\right\}$ commutes with $y$. This contradicts the assumption that for this case the center is the identity.

On the other hand, if $G_{i}$ and $G_{j}$ have only the identity in common, then $G$ has a representation as a permutation group of class $n-1$. Then by a theorem of Frobenius [2], $G$ has an invariant subgroup, $H$, necessarily Abelian since all subgroups of $G$ are, consisting of the permutations of degree $n$. Since $H$ is Abelian, its normalizer, which is $G$, is its centralizer. But then $H$ is in the center of $G$, again a contradiction, for $H$ cannot be the identity.

Since the theorem is true for groups whose order is prime, all of which satisfy the conditions and all of which are Abelian, the induction is complete.

\section{REFERENCES}

1. H. Zassenhaus, $A$ group-theoretic proof of a theorem of Maclagan-Wedderburn, Proceedings of the Glasgow Mathematical Association vol. 1 (1952) pp. 54-63.

2. G. Frobenius, Über anflösbare Gruppen, IV, Preuss. Akad. Wiss. Sitzungsber. (1901) pp. 1223-1225.

Iowa State College 